\title{
The Need for Standardized Tests to Evaluate the Reliability of Data Transport in Wireless Medical Systems
}

\author{
Helena Fernandez-Lopez ${ }^{1,3}$, José A. Afonso ${ }^{2,3}$, J.H. Correia ${ }^{2,3}$, \\ and Ricardo Simoes ${ }^{1,4,5}$ \\ ${ }^{1}$ Institute for Polymers and Composites IPC/I3N, University of Minho, 4800 Guimarães, \\ Portugal \\ ${ }^{2}$ Department of Industrial Electronics, University of Minho, 4800-058, Guimarães, Portugal \\ ${ }^{3}$ Centre Algoritmi, University of Minho, 4800-058, Guimarães, Portugal \\ ${ }^{4}$ Polytechnic Institute of Cávado and Ave, Campus do IPCA, 4750-810 Barcelos, Portugal \\ ${ }^{5}$ Life and Health Sciences Research Institute (ICVS), School of Health Sciences, \\ University of Minho, Campus de Gualtar, 4710-057 Braga, Portugal \\ \{hlopez, jose.afonso, higino.correia\}@dei.uminho.pt \\ rsimoes@dep.uminho.pt, rsimoes@ipca.pt
}

\begin{abstract}
Wireless medical systems are comprised of four stages, namely the medical device, the data transport, the data collection and the data evaluation stages. Whereas the performance of the first stage is highly regulated, the others are not. This paper concentrates on the data transport stage and argues that it is necessary to establish standardized tests to be used by medical device manufacturers to provide comparable results concerning the communication performance of the wireless networks used to transport medical data. Besides, it suggests test parameters and procedures to be used to produce comparable communication performance results.
\end{abstract}

Keywords: eHealth, medical data transport, communication performance assessment, standardized tests, wireless biomedical monitoring.

\section{Introduction}

For several decades, wearable patient monitors have been used in hospitals to continuously monitor ambulatory patients. Electrocardiogram (ECG), oxygen saturation $\left(\mathrm{SpO}_{2}\right)$, and other medical device information can be continuously monitored, in real time, even when patients are on the move. Until recently, these systems operated in dedicated spectrum bands and employed custom wireless technologies designed to optimize specific quality of service (QoS) requirements ${ }^{1}$. However, advances in radiofrequency (RF) and networking technologies have

\footnotetext{
${ }^{1}$ QoS refers to the ability of a network to deliver data reliably and timely. According to the IEEE Std. 11073-00101-2008, QoS requirements depend largely on the nature and criticality of the data being transported, and include reliability, latency, priority, and bandwidth. Within the scope of this work, the reliability of a data transport system refers to its ability to deliver the generated packets as measured by, for example, the packet reception ratio.
}

F. Martins, L. Lopes, and H. Paulino (Eds.): S-Cube 2012, LNICST 102, pp. 137-145, 2012.

(C) Institute for Computer Sciences, Social Informatics and Telecommunications Engineering 2012 
brought about new possibilities to healthcare providers and patients. For instance, pervasive networks based on the IEEE 802.11 protocols (commercialized as Wi-Fi) now approach the reliability of hardwired networks [1], allowing manufacturers to develop remote patient monitoring systems based on this protocol [2-4]. Besides, the rising costs in healthcare combined with significant developments in microelectronics, biomedical sensors and different classes of wireless networks decisively contributed to the increasing interest in e-Health systems ${ }^{2}$ [5], including those that employ wireless technologies to transport medical data. These systems have the potential to reduce healthcare costs while improving the quality of the healthcare services provided to a huge number individuals, particularly elderly, recovering and chronically-ill patients [6].

However, despite the clear benefits of wireless data transport, several issues apply. The wireless channel is an unpredictable and challenging medium. First, the radio spectrum is scarce. Consequently, some frequency bands are shared by different systems resulting in interference between neighboring devices. Moreover, QoS parameters, such as data transfer reliability and latency, may also fluctuate in response to changes in traffic, link quality and propagation conditions. Finally, security is difficult to implement since wireless communications are susceptible to eavesdropping [7].

The incorporation of standard RF technologies into medical devices for wireless data transport has motivated standard organizations to address key aspects related to the transport of medical data. The CEN ISO/IEEE 11073-00101-2008 standard ${ }^{3}$ [8] (hereinafter simply referred to as IEEE 11073-00101), which is part of the CEN ISO/IEEE 11073 family of standards, provides guidance for the utilization of point of care medical devices that exchange vital signs and other information using shared information technology infrastructure. This standard addresses technical and QoS performance requirements, besides security, privacy and coexistence issues. However, while requirements such as maximum end-to-end latency and bandwidth are clearly defined for various categories of medical data and scenarios, data transport reliability is only defined in qualitative terms, except for some equipment categories included in one specific scenario.

This limitation prevents the critical assessment of the suitability of emerging medical systems that employ wireless technologies to transport medical data. Besides, as there is no standardized evaluation test procedure that manufacturers should carry out, it is not possible to fairly compare the communication performance of different wireless medical systems. For instance, whereas some manufacturers assess the communication performance of a wireless network by measuring the total dropout proportion ${ }^{4}$ [9], some academic works opt for determining the packet reception ratio (PRR) or its statistical distribution $[10,11]$. Also, each study defines an arbitrary observation time.

This paper discusses the need of establishing standardized test procedures, to be used by medical device manufacturers, to provide comparable results about the communication performance of wireless networks used to transport medical data.

2 According to the World Health Organization (WHO), eHealth is defined as the combined use of electronic communication and information technology in the health sector.

3 CEN, ISO and IEEE stand for Comité Européen de Normalization or European Committee for Normalization, International Standards Organization, and Institute of Electrical and Electronics Engineers.

4 The total dropout proportion equals to the total dropout time divided by the total monitored time. 
Besides, it suggests test parameters and procedures that should be used to define standardized and repeatable tests that can produce comparable performance results.

\section{$2 \quad$ Related Work}

Sneha and Varshney [12] argue that the reliability of message delivery to healthcare professionals is the most critical requirement of patient monitoring. According to these authors, prioritization of different message types is vital to achieve high reliability. The reliability of message delivery on a wireless network can be evaluated through analytical models, simulations or experimental tests. In general, analytical models and simulations promise a fast evaluation that allows exploring the effect of relevant parameters and configurations. Although these performance evaluation approaches are steps towards obtaining insight into systems performance, an important further step is the execution of experimental tests [13]. However, if field tests are not standardized, it is difficult to compare the results obtained for different systems.

Differently from other works, this paper discusses the need for standardized test procedures for evaluating the reliability of wireless networks used to transport medical data. A similar effort in another area, the interoperability of information systems that exchange medical records, was recently carried out by the National Institute of Standards and Technology (NIST), in the USA, which elaborated several test procedures to improve the usability of Electronic Health Records [14].

\section{Wireless Medical Data Transport Regulation}

A wireless medical system can be described as being comprised of four stages [8], as shown in Fig. 1. The first stage includes the medical device, which can be an external device (e.g., a blood pressure monitor with wireless connectivity), a wearable or an implantable device. Data generated by devices are transported through multiple stages until reaching the patient or a health care provider.

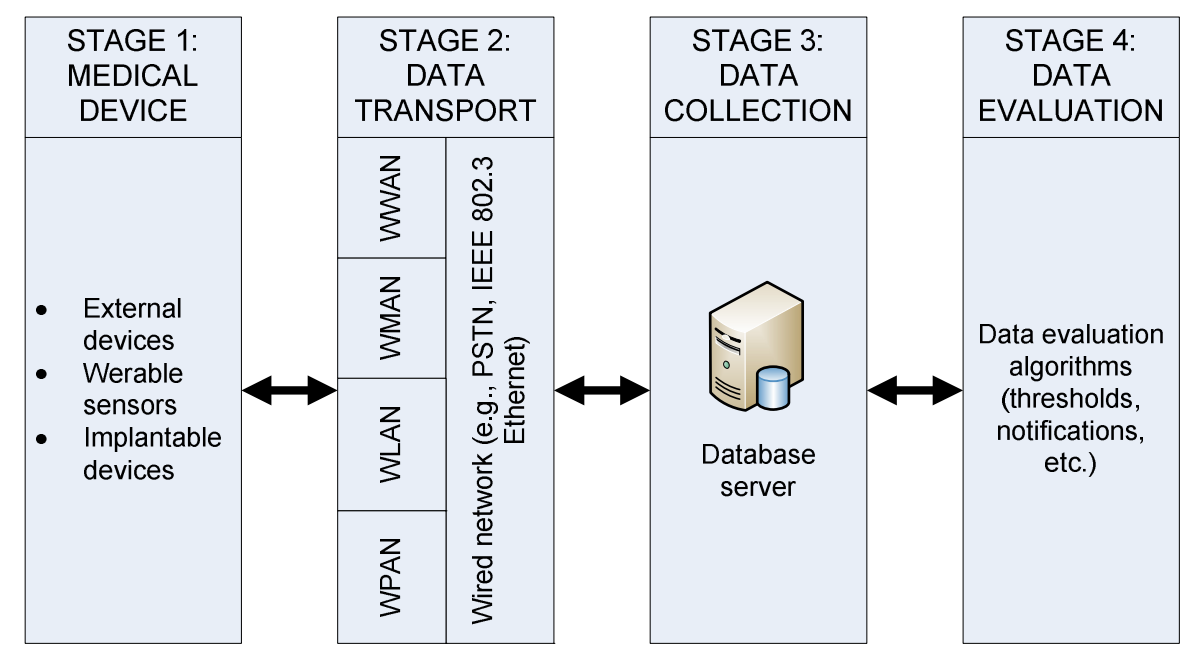

Fig. 1. Stages of a wireless medical system (adapted from [8]) 
Medical devices (stage 1 of Fig. 1) are regulated by federal government bodies. In most countries, these devices are classified into one of three or more classes. The regulatory requirements that manufacturers must comply with depend on the class which the device belongs. For instance, in the European Economic Area (EEA), medical devices can fall into one of the following classes: I, I sterile, I measure, IIa, IIb or III, with class III covering the highest risk products. Medical devices are regulated by three approach directives, depending on the classification of the device: Medical Device Directive 93/42/EEC [15]; Active Implantable Medical Device Directive 90/385/EEC [16]; or In-Vitro Diagnostic Medical Device Directive 98/79/EC [17]. These directives are in convergence to standards issued by the ISO, being the ISO 13485:2003, which defines the international quality system standards for medical devices, the most relevant [18]. These devices should also comply with product risk management, electromagnetic interference and compatibility (EMI/EMC) and usability regulations. Also, they must conform to local laws on personal data privacy and hardware disposal (Restriction of Hazardous Substances Directive or RoHS).

Whereas stage 1 of wireless medical systems is strictly regulated, the other stages are not. Current standardized technologies included in stage 2 were not designed to transport medical data and to support the QoS requirements that this transport involves. According to [8], such systems might not be considered a medical device when operating under typical conditions. Connectivity, including wireless connectivity based on standard-based technologies, is not considered on medical regulatory documents, but instead in nonmedical standards (e.g., IEEE standards) and nonmedical consortium agreements (e.g., Wi-Fi Alliance and ITU). The regulation within stage 2 would be hard to accomplish because data transport involves complex combinations of distinct technologies that include wireless personal area networks (WPANs), wireless local networks (WLANs), wireless metropolitan area networks (WMANs) and wireless wide area networks (WWANs). Data storage in stage 3 shares the same difficulties as stage 2 , as it is defined by several nonmedical standards. Additionally, in several cases, stage 4 merges with stage 3 as it is based on applications that include data storage and analysis.

The CEN ISO/IEEE 11073 family of standards, which was developed in coordination with other standards development organizations, including IEEE 802 committee, $\mathrm{IHTSDO}^{5}, \mathrm{HL}^{6}$ and $\mathrm{DICOM}^{7}$, aims to provide real-time, plug-and-play ${ }^{8}$ interoperability between point-of-care medical devices. Additionally, it aims to promote the efficient exchange of care device data. The IEEE 11073-00101 standard covers several application

5 The International Health Terminology Standards Development Organization (IHTSDO) is a not-for-profit association that develops and promotes use of SNOMED CT, a multilingual health care clinical terminology.

6 The Health Level Seven International (HL7) is a global authority on standards for interoperability of health information technology.

7 The Digital Imaging and Communications in Medicine (DICOM) is a standard designed to ensure the interoperability of systems that deal with medical images and derived structured documents as well as to manage related workflow.

8 Plug-and-play interoperability means that the user does not need to do any action, apart from connecting the device, to allow it to communicate data as defined. 
use cases and considers potential applications for standard-based communication technologies, including cellular, IEEE 802.11, IEEE 802.15.4 and ZigBee protocols. Additionally, it defines QoS parameters, namely reliability, latency, priority, and bandwidth requirements, for some data classes of interest, as shown in Table 1. These parameters may be used in prioritizing and securing categories of data generated by devices that share a wireless network. As shown, real-time alarms and alerts should have the highest reliability and priority, whereas real-time patient state change messages and real-time reminders shall have highest reliability as well, but medium priority. Real-time waveform data and other real-time physiologic parameters, such as episodic blood pressure (BP) and heart rate (HR), shall have high reliability and priority.

Table 1. QoS requirements for some categories of medical device data (adapted from [8])

\begin{tabular}{|l|l|l|l|}
\hline Data category & Reliability $^{\left({ }^{*}\right)}$ & Latency & Priority $^{(*)}$ \\
\hline $\begin{array}{l}\text { Alerts/alarms (real- } \\
\text { time) }\end{array}$ & $\begin{array}{l}\text { highest/ } \\
\text { essential }\end{array}$ & $\begin{array}{l}<500 \text { ms from the wireless } \\
\text { sensor to the gateway to the } \\
\text { wired network } \\
<3 \mathrm{~s} \text { communication latency }\end{array}$ & $\begin{array}{l}\text { highest/ } \\
\text { essential }\end{array}$ \\
\hline $\begin{array}{l}\text { Patient state change } \\
\text { (real-time) }\end{array}$ & $\begin{array}{l}\text { highest/ } \\
\text { essential }\end{array}$ & $<3 \mathrm{~s}$ communication latency & medium \\
\hline Reminder (real-time) & $\begin{array}{l}\text { highest/ } \\
\text { essential }\end{array}$ & $<3 \mathrm{~s}$ communication latency & medium \\
\hline Waveforms (real-time) & high & $\begin{array}{l}<3 \mathrm{~s} \text { to central station } \\
<7 \mathrm{~s} \text { for telemetry to in- } \\
\text { room monitor }\end{array}$ & high \\
\hline $\begin{array}{l}\text { Physiologic parameters } \\
\text { (e.g., episodic BP, HR, } \\
\text { SpO }\end{array}$ & high temp.) (real-time) & $\begin{array}{l}<10 \mathrm{~s} \text { to central station } \\
<3 \mathrm{~s} \text { communication latency } \\
\text { from monitor to clinician }\end{array}$ & high \\
\hline
\end{tabular}

(*) Ratings: low, medium, high and highest/essential.

As shown in Table 1, whereas maximum latency values are specified by the IEEE 11703 -for each category of data, reliability is only qualitatively specified (highest/essential, high, medium and low). The only exceptions are the maximum data losses allowed for some data categories included in the UC2 scenario, single cardiac patient in hospital, which are presented in Table 2.

Table 2. Data loss values for some data categories included in the UC2 scenario (adapted from [8])

\begin{tabular}{|c|c|c|}
\hline Device & Data category & Data loss \\
\hline $\begin{array}{l}\text { PWD - Patient-worn } \\
\text { device }\end{array}$ & 1 ECG vector & $\begin{array}{l}<5 \text { seconds per event } \\
<4 \text { events per hour }\end{array}$ \\
\hline $\begin{array}{l}\text { Wireless vital signs } \\
\text { monitor }\end{array}$ & $\begin{array}{l}\text { PDS - Parameter data service } \\
\left(\mathrm{BP}, \mathrm{HR}, \mathrm{SpO}_{2} \text {, respiration) }\right. \\
\text { Alarm data services }\end{array}$ & $\begin{array}{l}<5 \text { seconds per event } \\
<4 \text { events per hour } \\
<1 \text { second }\end{array}$ \\
\hline
\end{tabular}

Finally, the IEC 80001-1:2010 [19] defines roles, responsibilities and activities that are necessary for risk management of IT-networks incorporating medical devices to 
address safety, effectiveness and data and system security. However, it applies after the medical device has been acquired by the organization and does not define minimum performance parameters.

\section{Assessment of the Communication Performance of Networks Used to Transport Medical Data}

In a recent work, the authors have evaluated the communication performance of a prototype remote patient monitoring system consisting of six ZigBee-based ECG devices [20]. Fig. 2 presents the per 2-second PRR values measured for a specific wireless ECG sensor device over a period of 16.7 hours. This device was two hops away from the personal area network (PAN) coordinator and achieved a mean PRR of 0.99 . However, as shown in Fig. 2, during a contention period of approximately 30 minutes, the PRR values varied considerably, reaching a minimum of 0.6 , which, despite the good mean PRR, might be unacceptable for certain scenarios.

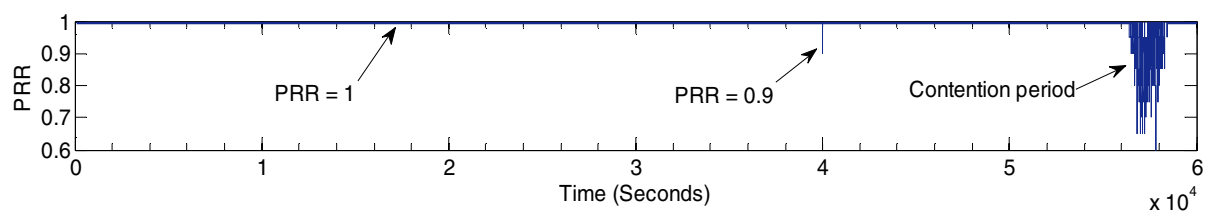

Fig. 2. PPR values for a ZigBee-based ECG device measured over a period of 16.7 hours

As illustrated, test results expressed using mean values alone (e.g., mean PRR, mean dropout duration, mean time between dropouts and total dropout proportion) are unable to provide complete information about the reliability of a wireless network. However, mean values are often presented by several studies, including those provided by medical device manufacturers. The occurrence of transitory low communication performance periods, as the one exemplified on Fig. 2, may not become evident to a clinical engineer in charge of evaluating the performance of a wireless medical device if PRR values along the time are not provided. Besides, if evaluation methods are not standardized, it may not be possible to fairly compare the communication performance of different systems.

Consequently, it is necessary to define standardized tests that can provide, to all involved stakeholders, essential information about the communication performance of the wireless networks used to transport medical data. In a first approach toward solving the identified problem, it is suggested that performance assessment tests include the general aspects presented in Table 3. They contain different wireless channel conditions, mobility scenarios, scalability issues, and failure conditions. Also, it is recommended using a test platform that guarantees controlled conditions in order to assure repeatability. Such a platform was presented by Hu et al., from National ICT Australia and University of Queensland [21], who evaluated the performance of specific IEEE 802.11a-based mesh backhaul radios. The referred platform provided an environment free of interference. Besides, it allowed researchers control the quality of all links and simulate link and device failures. 
Table 3. General procedures suggested for performance assessment of wireless networks used to transport medical data

\begin{tabular}{|l|l|}
\hline Aspects & Procedure \\
\hline $\begin{array}{l}\text { Wireless channel } \\
\text { conditions }\end{array}$ & $\begin{array}{l}\text { Ideal propagation: consider free-space path loss determined using } \\
\text { the maximum range specified for the patient-worn device. }\end{array}$ \\
\cline { 2 - 2 } Mobility & $\begin{array}{l}\text { Non-ideal propagation conditions: consider additional attenuation } \\
\text { caused by different effects (e.g. multipath, interference and } \\
\text { obstruction caused by large objects and structures). }\end{array}$ \\
\hline Scalability & $\begin{array}{l}\text { Consider one or more mobile patient-worn devices which move } \\
\text { from one parent device/sink to another one. }\end{array}$ \\
\hline $\begin{array}{l}\text { Backhaul device } \\
\text { failure, link failure } \\
\text { and connection } \\
\text { loss }\end{array}$ & $\begin{array}{l}\text { Add patient worn devices until the maximum number of devices } \\
\text { supported. Consider situations where devices should associate to the } \\
\text { same or to different parent devices/sinks. Also, for multihop } \\
\text { networks, consider a crescent number of hops. }\end{array}$ \\
$\begin{array}{l}\text { Measure the time necessary to reassociate to a new parent } \\
\text { device/sink in case of backhaul device or link failure. Consider } \\
\text { reliability requirements of different data categories and distinct } \\
\text { reconfiguration actions. }\end{array}$ \\
\cline { 2 - 2 } $\begin{array}{l}\text { In case the device moves to an area without connectivity, verify if } \\
\text { actions taken are acceptable (e.g., alerts to care givers and patient). }\end{array}$ \\
\hline
\end{tabular}

In order to correctly evaluate the communication performance of a medical device, it is suggested keeping track of the following QoS parameters:

- PRR measured over time using a temporal window of appropriate length for each active patient worn-device and medical data category (refer to Table 2 of [8] for the comprehensive list of medical data categories);

- overall and per-active patient-worn device average dropout;

- end-to-end latency associated to each message and the mean and maximum end-to-end latency values for each medical data category;

- jitter incurred by periodic traffic with constant packet size; and

- bandwidth required per active patient-worn device.

As stated above, it is suggested measuring the PRR over a small time window in order to capture transitory effects that compromise the system performance. For instance, in carrier sense multiple access with collision avoidance (CSMA-CA) based networks transitory contention conditions between devices may result in temporary message losses generated by contending devices. Besides, reconfigurations, such as rate changes from multi-rate mechanisms used to adapt the transmission rate to the channel condition in IEEE 802.11-based networks, can temporarily impact the performance of the active devices involved.

During the lifetime of the equipment, it is essential that field tests are performed. Ideally, the medical system would incorporate a test module capable of acquiring data that would alert clinical staff of any changes in the reliability of the system. Also, it would provide clinical engineers means to execute field tests and, in case of any 
trouble, supply the detailed information needed to diagnose the problem. In case of routinely field tests required for system evaluation, these tests should last long enough to capture most variations in environmental conditions. For instance, in a hospital or nursing home scenario, a field test may last one week to capture events that can influence the RF environment. These events would include the increased influx of visitors during the weekends which, in general, have negative effects on link conditions.

\section{Conclusions}

This paper discusses the lack of quantitative reliability parameters to evaluate the communication performance of medical systems that employ wireless communication technologies. The IEEE 11073-00101, which provides general guidance for the use of standard RF technologies to transport medical data, establishes maximum latency values for each data type. A similar approach is needed for reliability.

Moreover, it demonstrates the need for standardized and repeatable tests to be used by medical device manufacturers to produce comparable communication performance evaluation results regarding medical systems that use wireless networks to transport data. In order to provide an initial contribution to this field, it suggests general test parameters and procedures. The QoS parameters to be measured include the instantaneous and mean PRR per-active patient-worn device and the bandwidth required by each device. The general procedures include measurements performed under different channel conditions and mobility scenarios. Also, it suggests addressing scalability and different failure conditions, such as backhaul device failure, link failure and connection loss.

Future work includes the communication performance assessment of a prototype wireless medical system using the guidelines proposed in order to evaluate the suggested approach.

Acknowledgments. Authors would like to thank Mr. Teófilo Leite, Mr. Nélson Brito and Ms. Teresa Moura, from Hospital Privado de Guimarães, for the encouragement and support. This work has been supported by the Portuguese Foundation for Science and Technology (FCT), Lisbon, through the $3^{\circ}$ Quadro Comunitário de Apoio, the POCTI and FEDER programs, the MIT-Portugal program, project PEstC/CTM/LA0025/2011 (Strategic Project - LA 25 - 2011-2012), and the FCT grant SFRH/BD/39408/2007. AAL4ALL project, co-financed by the European Community Fund through COMPETE - Programa Operacional Factores de Competitividade. Clinical and financial support for this study has been provided by Grupo AMI Assistência Médica Integral (Casa de Saúde Guimarães, SA), Portugal, under the partnership established between this healthcare company and the University of Minho. We also acknowledge useful discussions with staff from the Hospital de Braga. 


\section{References}

1. Baker, S.D., Hoglund, D.H.: Medical-Grade, Mission-Critical Wireless Networks (Designing an Enterprise Mobility Solution in the Healthcare Environment). IEEE Engineering in Medicine and Biology Magazine 27, 86-95 (2008)

2. Allyn, W.: Acuity Central Monitoring Station, http: / / www . welchallyn.com/wafor/hospitals/emr_connectivity/ solutions_WACS.htm

3. Philips Healthcare. Philips Intellivue Telemetry System $x 40$, http: / /www.healthcare.philips.com/main/products/ patient_monitoring/products/intellivue_mx40/index.wpd

4. Intelesens. Aingeal Patched-based Vital Signs, http: / / www. intelesens.com/pdf / aingealdatasheet.pdf

5. Kyriacou, E., et al.: m-Health e-Emergency Systems: Current Status and Future Directions. IEEE Antennas and Propagation Magazine 49, 216-231 (2007)

6. Ekeland, A.G., Bowes, A., Flottorp, S.: Efectiveness of telemedicine: A systematic review of reviews. International Journal of Medical Informatics 79, 736-771 (2010)

7. Goldsmith, A.: Wireless Communications. Cambridge University Press, New York (2005)

8. IEEE 11073-00101-2008 Health Informatics - PoC Medical Device Communication - Part 00101: Guide - Guidelines for the Use of RF Wireless Technology, 1-99 (2008)

9. Allyn, W.: FlexNet for 802.11a life-critical wireless networks, http: / / www. welchallyn.com/products/en-us / $\mathrm{x}-16$-vo-96-1234190869014.htm

10. Ko, J., Gao, T., Terzis, A.: Empirical Study of a Medical Sensor Application in an Urban Emergency Department. In: Proceedings of the Fourth International Conference on Body Area Networks, ICST, pp. 1-8. ICST, Belgium (2009)

11. Chipara, O., Lu, C., Bailey, T.C., Roman, G.-C.: Reliable Patient Monitoring: A Clinical Study in a Step-down Medical Unit. Technical report, Washington University in Saint Louis, Saint Louis (2009)

12. Sneha, S., Varshney, U.: Enabling ubiquitous patient monitoring: Model, decision protocols, opportunities and challenges. Decision Support Systems 46, 606-619 (2009)

13. Ali, M., et al.: Medium Access Control Issues in Sensor Networks. ACM Computer Communication Review 36, 33-36 (2006)

14. National Institute of Standards and Technology (NIST), http: / / www.nist.gov/

15. Council Directive 93/42/EEC of 14 June 1993 concerning medical devices (1993)

16. Council Directive 90/385/EEC of 20 June 1990 on the approximation of the laws of the Member States relating to active implantable medical devices (1990)

17. Directive 98/79/EC of the European Parliament and of the Council of 27 October 1998 on in vitro diagnostic medical devices (1998)

18. ISO 13485:2003 Medical devices - Quality management systems - Requirements for regulatory purposes, $1-57$ (2003)

19. IEC 80001-1:2010 Application of risk management for IT-networks incorporating medical devices - Part 1: Roles, responsibilities and activities, 1-70 (2010)

20. Fernandez-Lopez, H., Afonso, J.A., Correia, J.H., Simoes, R.: HM4All: A vital Signs Monitoring System based in Spatially Distributed ZigBee Networks. In: 4th International Conference on Pervasive Computing Technologies for Healthcare, pp. 1-4 (2010)

21. $\mathrm{Hu}, \mathrm{P}$., et al.: Evaluation of commercial wireless mesh technologies in a public safety context: Methodology, analysis and experience. In: IEEE 7th International Conference on Mobile Ad Hoc and Sensor Systems, pp. 606-611 (2010) 